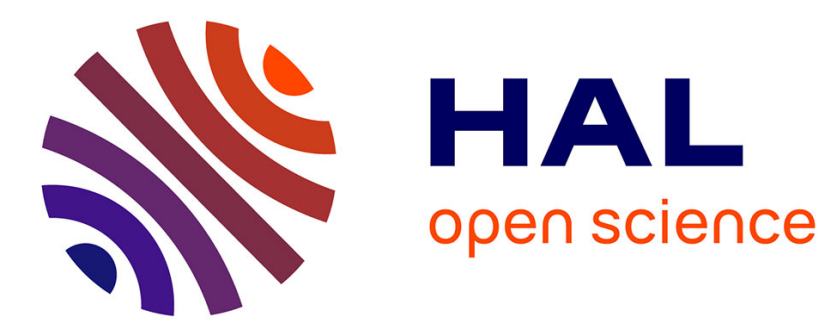

\title{
De la maladie rénale chronique à la transplantation rénale
}

Johanna Rabiller, Sébastien Faure

\section{To cite this version:}

Johanna Rabiller, Sébastien Faure. De la maladie rénale chronique à la transplantation rénale. Actualités Pharmaceutiques, 2019, 58, pp.20 - 25. 10.1016/j.actpha.2019.01.014 . hal-03486080

\section{HAL Id: hal-03486080 \\ https://hal.science/hal-03486080}

Submitted on 20 Dec 2021

HAL is a multi-disciplinary open access archive for the deposit and dissemination of scientific research documents, whether they are published or not. The documents may come from teaching and research institutions in France or abroad, or from public or private research centers.
L'archive ouverte pluridisciplinaire HAL, est destinée au dépôt et à la diffusion de documents scientifiques de niveau recherche, publiés ou non, émanant des établissements d'enseignement et de recherche français ou étrangers, des laboratoires publics ou privés.

\section{다)(1) $(5$}

Distributed under a Creative Commons Attribution - NonCommercial| 4.0 International 
Version of Record: https://www.sciencedirect.com/science/article/pii/S0515370019300436

Manuscript_73852782d295bd1188efbcb2f09bec2a

Dochead dossier

Sous-dochead La greffe rénale

\section{De la maladie rénale chronique à la transplantation rénale}

Johanna Rabiller ${ }^{\mathrm{a}}$

Pharmacienne adjointe

Sébastien Faure ${ }^{\mathrm{b}, *}$

Professeur des Universités

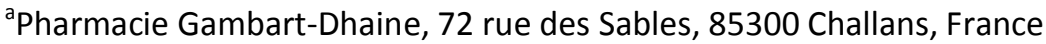

${ }^{\mathrm{b}}$ Faculté de santé, Département pharmacie, Université d'Angers, 16 boulevard Daviers, 49045 Angers, France

*Auteur correspondant.

Adresse e-mail : sebastien.faure@univ-angers.fr (S. Faure).

Résumé

La transplantation rénale constitue le meilleur traitement de suppléance de l'insuffisance rénale terminale avec une survie et une qualité de vie améliorées, une moindre morbidité cardiovasculaire et un coût de traitement inférieur après la première année. Malgré les progrès, la technique de greffe se heurte toujours à un obstacle majeur, la question du rejet.

(C) 2019

Mots clés - don d'organes ; greffe ; insuffisance rénale chronique ; maladie rénale ; transplantation Summary à venir

(c) 2018

Keywords à venir

La prévalence des maladies rénales est en augmentation constante en France et dans le monde, principalement du fait du vieillissement de la population [1].

La classification des néphropathies repose généralement sur les données histologiques de la biopsie rénale :

- les néphropathies glomérulaires sont caractérisées par des lésions du glomérule (la plus fréquente est la maladie de Berger chez l'adulte jeune);

- les néphropathies tubulo-interstitielles présentent des lésions qui touchent essentiellement l'espace interstitiel et la partie tubulaire ;

- les néphropathies vasculaires concernent les artères du rein ;

(C) 2019 published by Elsevier. This manuscript is made available under the CC BY NC user license https://creativecommons.org/licenses/by-nc/4.0/ 
- d'autres pathologies rénales, comme les tumeurs du rein ou les anomalies congénitales du développement rénal, font partie de cette classification [2].

Les néphropathies résultent de diverses agressions, notamment les anomalies génétiques, les anomalies congénitales du développement de l'appareil urinaire, les obstacles, les infections, certains médicaments (dextrans de faible poids moléculaire, anticancéreux et immunomodulateurs comme le méthotrexate ou le cyclophosphamide, antibiotiques tels que les cyclines ou la colimycine) et les toxiques. Les maladies hématologiques (lymphome, myélome) et les pathologies vasculaires dont l'hypertension artérielle (HTA) et la sténose bilatérale des artères rénales sont également à prendre en compte. Enfin, il est important de noter l'intervention d'un nombre non négligeable d'affections auto-immunes [3]. Par exemple, les maladies tubulo-interstitielles sont généralement causées par des uropathies chroniques, par obstruction chronique des voies excrétrices (adénome prostatique, lithiase) ou la prise prolongée de certains médicaments (lithium, diurétiques hypokaliémants...). En revanche, les causes les plus fréquentes de néphropathies vasculaires sont I'HTA, la sténose athéromateuse des artères rénales, l'embolie générée par la prise d'un traitement anticoagulant ou fibrinolytique... [2]. Toutes ces atteintes peuvent conduire à une insuffisance rénale (IR).

\section{T1 L'insuffisance rénale}

TEG1 L'insuffisance rénale correspond à l'altération des deux reins qui ne filtrent plus correctement le sang. La maladie est dite "aiguë" si le dysfonctionnement est transitoire et réversible, chronique si la destruction est irréversible, sans possibilité de guérison [4].

TEG1 L'insuffisance rénale aiguë (IRA) est un syndrome qui résulte d'une détérioration rapide (en quelques heures ou jours) de la fonction excrétrice des reins, le plus souvent avec oligurie (diminution du volume des urines). L'incapacité d'élimination, conséquence de la diminution brutale du débit de filtration glomérulaire (DFG), se traduit par un syndrome de rétention azotée (augmentation de la créatinémie et azotémie) et des troubles liés à un déséquilibre du bilan hydroélectrolytique (hyperkaliémie, hyperhydratation, acidose métabolique). L'IRA est potentiellement réversible, soit spontanément, soit par traitement de la cause.

TEG1 L'insuffisance rénale chronique (IRC), à l'inverse, ne régresse pas. Se manifestant par une diminution progressive du DFG, elle résulte d'une destruction anatomique irréversible des néphrons, ce qui se traduit par une altération des différentes fonctions rénales (fonctions d'épuration, d'excrétion, de régulation et fonctions endocrines).

TEG1 L'IRC est la conséquence, dans $80 \%$ des cas, de néphropathies primitives relatives à des lésions glomérulaires (glomérulonéphrites primitives), interstitielles (néphrites interstitielles) ou vasculaires (néphropathies vasculaires). Toutefois, elle est aussi induite par d'autres facteurs comme le diabète et l'HTA. La fréquence des néphropathies diabétiques et vasculaires est en progression. D’après une étude de 2003, dix ans après le début d'un diabète, un tiers des patients développe une insuffisance rénale dont $6 \%$ à un stade avancé. Cependant, l'amélioration constante de la prise en charge du diabète a pu modifier ces données [5]. Enfin, les néphropathies héréditaires comme la polykystose rénale ou les séquelles d'une IRA peuvent expliquer l'apparition d'une IRC. Dans le cas où l'IRC est installée depuis plus de trois mois consécutifs, il est question de maladie rénale chronique (MRC).

\section{T1 La maladie rénale chronique}


TEG1 La MRC est une affection fréquente et mésestimée en population générale, constituant ainsi un enjeu majeur de santé publique.

TEG1 En France, les connaissances épidémiologiques la concernant restent faibles. Il a pourtant été estimé, en 2012, que la prévalence de la MRC était de $30 \%$ chez les patients de plus de 70 ans [6]. L'étude Epiran, débutée en 2004, a été réalisée en Lorraine dans le but d'évaluer l'incidence de l'IRC en population générale et de décrire les caractéristiques des patients concernés. Près de 50000 dosages de créatinine ont été déclarés, pour près de 4400 patients. Parmi eux, 631 étaient des sujets incidents. L'estimation de l'IRC a ainsi été portée à $1 \%$ habitants $(1,3 \%$ chez les hommes et $0,7 \%$ chez les femmes). Les patients étaient très âgés ( 77 ans en moyenne) et atteints de multiples pathologies (34\% étaient diabétiques, $23 \%$ insuffisants cardiaque). Aussi, le nombre de personnes concernées serait en augmentation, en particulier à cause de l'accroissement des néphropathies diabétiques, hypertensives et vasculaires [7].

TEG1 La MRC reste grave puisque le risque de progression vers l'insuffisance rénale terminale (IRT) et une morbidité cardiovasculaire accrue est potentialisé [5].

\section{T1 Facteurs de risque}

TEG1 Les éléments sociodémographiques et cliniques qui invitent à dépister une MRC sont nombreux (encadré 1).

TEG1 La présence d'un ou plusieurs de ces facteurs chez un individu incite à estimer la fonction rénale.

\section{Encadré 1. Facteurs de risque de maladie rénale chronique}

- Âge $>65$ ans

- Sexe masculin

- Peau noire

- Conditions socio-économiques précaires

- Hypertension artérielle

- Diabète, obésité, syndrome métabolique

- Maladies cardiovasculaires

- Uropathie, malformation urinaire, lithiase rénale

- Maladies auto-immunes

- Dysglobulinémies monoclonales

- Hépatopathie

- Médicaments toxiques

- Antécédents familiaux de maladies rénales

- Antécédents d'insuffisance rénale aiguë 
- Grossesse

- Poids de naissance $<2,5 \mathrm{~kg}$

\section{T1 Signes cliniques et biologiques}

TEG1 Le diagnostic souvent tardif de la MRC s'explique par la capacité d'adaptation des néphrons "sains" restants qui s'hypertrophient sous l'influence de facteurs de croissance. Ces derniers permettent de maintenir les capacités d'excrétion du rein et d'assurer l'équilibre hydroélectrolytique de l'organisme jusqu'à un stade avancé de la MRC.

TEG1 Malgré cette adaptation, un syndrome urémique s'installe et se caractérise par une rétention de métabolites toxiques provenant principalement du métabolisme protéique (accumulation dans le sang d'urée, de créatinine, etc., par diminution de l'élimination rénale).

TEG1 Les symptômes cliniques liés à la MRC sont variables et principalement dus au syndrome urémique : fatigue (en particulier à l'effort, essoufflement, difficultés de concentration, troubles du sommeil), envie fréquente d'uriner (surtout la nuit), manque d'appétit et dégoût vis-à-vis de certains aliments (de la viande en raison de l'accumulation de l'urée dans le sang), peau sèche et prurigineuse, crampes et œdèmes.

TEG1 Sur le plan biologique, la MRC se caractérise par :

- une augmentation de la créatininémie, de l'urée sanguine, de l'uricémie, de la kaliémie et de la phosphorémie ;

- une diminution de la forme active de la vitamine $D$ due à un défaut d'hydroxylation en $C 1$ générant ainsi une diminution de l'absorption du calcium et donc une hypocalcémie ;

- une anémie hémolytique générée par une diminution de la synthèse d'érythropoïétine (EPO) et la présence d'hémolyse ;

- une HTA générée par une augmentation de la synthèse de la rénine et une rétention hydrosodée.

TEG1 Des complications osseuses et vasculaires peuvent être générées par l'hypocalcémie et l'augmentation de la phosphorémie. En effet, l'ostéomalacie liée à la non-synthèse de vitamine $D$ et I'hyperdestruction osseuse due à une hyperparathyroïdie secondaire sont responsables d'une atteinte osseuse mixte appelée ostéodystrophie rénale. La libération de calcium provoquée par la destruction des os peut également provoquer des calcifications vasculaires diffuses. Les calcifications précoces des artères augmentent notamment le risque de complications cardiovasculaires.

\section{T1 Diagnostic}

TEG1 La MRC est définie, indépendamment de sa cause, par la présence d'au moins un marqueur d'atteinte rénale ou d'une baisse du DFG au-dessous de $60 \mathrm{~mL} / \mathrm{min} / 1,73 \mathrm{~m}^{2}$ durant plus de trois mois consécutifs. Les marqueurs d'atteinte rénale peuvent être les suivants :

- micro-albuminurie : $20-200 \mu \mathrm{g} / \mathrm{min}$ ou $30-300 \mu \mathrm{g} / 24 \mathrm{~h}$ ou rapport albuminurie/créatininurie

$>2 \mathrm{mg} / \mathrm{mmol}$;

- protéinurie : $>300 \mathrm{mg} / 24 \mathrm{~h}$ ou rapport protéinurie/créatininurie $>200 \mathrm{mg} / 24 \mathrm{~h}$;

- hématurie pathologique : globules rouges $>10 / \mathrm{mm}^{3}$ ou $10000 / \mathrm{mL}$; 
- leucocyturie pathologique : globules blancs $>10 / \mathrm{mm}^{3}$ ou $10000 / \mathrm{mL}$;

- anomalies morphologiques à l'échographie rénale : asymétrie de taille, contours bosselés, reins de petite taille ou gros reins polykystisques...

\section{T1 Classification}

TEG1 La classification internationale de la MRC (tableau 1) permet de préciser le stade évolutif de la maladie en fonction du DFG [6], mais aussi de refléter le niveau de gravité et d'adopter des mesures de prévention adaptées à chacun des stades.

TEG1 Les principales mesures de prévention reposent tout d'abord sur le dépistage et la réduction des facteurs de risque susceptibles de générer une MRC (alimentation, exposition professionnelle, médicaments néphrotoxiques...), mais aussi le dépistage des pathologies pouvant initier une MRC (diabète, HTA, infections systémiques). Les autres mesures préventives consistent à diagnostiquer et traiter la MRC le plus rapidement possible afin d'éviter sa progression aux stades supérieurs, mais aussi de prendre en charge les comorbidités. La préservation du capital veineux (en vue d'une éventuelle hémodialyse), la restriction protidique et le vaccin contre I'hépatite $B$ font partie des moyens mis en œuvre pour préserver le rein déjà atteint.

Tableau 1. Classification internationale de la maladie rénale chronique.

\begin{tabular}{lll}
\hline Stade & $\begin{array}{l}\text { Débit de filtration } \\
\text { glomérulaire (DFG) } \\
\left(\mathbf{m L} / \mathbf{m i n} / \mathbf{1}, \mathbf{7 3} \mathbf{~ m}^{\mathbf{2}}\right)\end{array}$ & Description \\
\hline $\mathbf{1}$ & 90 & $\begin{array}{l}\text { Maladie rénale chronique }^{1} \\
\text { avec DFG normal ou augmenté }\end{array}$ \\
\hline $\mathbf{2}$ & $60-89$ & $\begin{array}{l}\text { Maladie rénale chronique } \\
\text { avec DFG légèrement diminué }\end{array}$ \\
\hline $\mathbf{3}$ & $59-30$ & $\begin{array}{l}\text { Insuffisance rénale chronique } \\
\text { modérée }\end{array}$ \\
\hline $\mathbf{4}$ & $15-29$ & $\begin{array}{l}\text { Insuffisance rénale chronique } \\
\text { sévère }\end{array}$ \\
\hline $\mathbf{5}$ & $<15$ & $\begin{array}{l}\text { Insuffisance rénale chronique } \\
\text { terminale }\end{array}$ \\
\hline 5D & Patient dialysé \\
\hline 5T & & Patient transplanté \\
\hline 1
\end{tabular}

${ }^{1}$ En présence de marqueurs d'atteinte rénale.

En cas de DFG $<60 \mathrm{~mL} / \mathrm{min} / 1,73 \mathrm{~m}^{2}$ chez un sujet n'ayant pas d'antériorité, il convient de répéter l'examen dans les deux semaines, en sachant que, lors de l'interprétation du résultat, la variabilité biologique et analytique de la créatine plasmatique est de plus ou moins $5 \%$.

Sur 2 colonnes près de son appel

\section{T1 La transplantation rénale}

TEG1 La première expérimentation de greffe rénale est réalisée au début du $x x^{e}$ siècle, d'abord de l'animal vers l'animal, puis de l'animal vers l'homme et, enfin, de l'homme vers l'homme.

TEG1 La première transplantation rénale d'un homme vers un autre est effectuée en Russie en 1933, par Serguey Voronoy. Ce dernier greffe le rein d'un homme décédé au niveau de l'aine d'une jeune malade âgée de 26 ans qui décédera quatre jours après. 
TEG1 Après la Seconde Guerre mondiale, la transplantation rénale focalise de nouveau l'attention de plusieurs équipes chirurgicales, aux États-Unis et en France notamment. Le greffon est, cette fois, placé dans l'abdomen. La technique chirurgicale progresse, la survie du patient greffé s'améliore et atteint parfois plusieurs mois.

TEG1 En 1952, la greffe rénale prend un tournant majeur en France, avec la première tentative à partir d'un donneur vivant : une mère souhaite que l'un de ses reins soit greffé à son fils de 17 ans qui vient de perdre son unique rein dans un accident. L'opération, réalisée à l'hôpital Necker par l'équipe du professeur Jean Hamburger, est un succès même si, 21 jours après, le jeune homme décède.

TEG1 Le rein représente l'organe le plus demandé par les malades en attente de greffe d'organes avec une hausse de $87 \%$ sur 20 ans, devant le foie, le cœur, le poumon et le pancréas. Depuis 1959, plus de 70000 greffes rénales ont été enregistrées (3 546 en 2018) alors que le nombre estimé de malades porteurs d'un greffon rénal est d'environ 38000 [8].

\section{T1 Indications de la transplantation rénale}

TEG1 La transplantation rénale peut être envisagée chez tous les patients présentant une MRC au stade 5 , qu'ils bénéficient déjà d'une dialyse ou que celle-ci soit imminente (la greffe est alors dite "préemptive").

TEG1 Certaines conditions sont toutefois requises : il faut que le patient en exprime la volonté, que les risques encourus n'excèdent pas les bénéfices escomptés et qu'il n'y ait pas de contre-indication. TEG1 La seule contre-indication absolue est l'existence d'un cancer métastasé. Les contreindications temporaires sont les infections non guéries et les antécédents de cancer. L'existence d'une sérologie positive pour le virus de l'immunodéficience humaine (VIH) ne constitue plus, depuis peu, une contre-indication car les résultats observés, en particulier en France, sont excellents. II reste néanmoins quelques contre-indications relatives : l'insuffisance cardiaque sévère, une coronopathie ou une maladie psychiatrique non stabilisée [5].

\section{T1 L'attribution des reins}

TEG1 Les reins greffés peuvent provenir de donneurs décédés ou vivants (respectivement, $84 \%$ et $16 \%$ en 2018) (encadré 2). La mort engendre toujours la destruction totale et irréversible des fonctions de l'encéphale, à savoir l'ensemble formé par le cerveau, le cervelet et le tronc cérébral. Cependant, il existe plusieurs processus biologiques et physiologiques y conduisant. Le donneur peut être en état de mort encéphalique ou décédé par arrêt cardiaque.

\section{Encadré 2. Le cas particulier des mineurs}

Jusqu'à 18 ans, ce sont les parents ou les tuteurs légaux qui prennent la décision de prélever. Cependant, si l'enfant avait fait part d'une volonté personnelle vis-à-vis du don d'organes, elle est prise en compte dans la majorité des cas [9].

Sur 1 colonne

TEG1 L'état de mort encéphalique est déclaré lorsque le cerveau n'est plus irrigué ni oxygéné par le sang, en cas de traumatisme crânien ou d'accident vasculaire cérébral (AVC) notamment. Lorsqu'un prélèvement est envisageable, l'activité cardiaque et la respiration peuvent être maintenues 
artificiellement durant quelques heures. Irrigués en sang et en oxygène, les principaux organes demeurent ainsi en bon état fonctionnel, le temps que l'autorisation de prélever soit donnée/confirmée, puis que le prélèvement soit réalisé [9].

TEG1 Un programme de prélèvement sur donneur décédé après arrêt cardiaque a été lancé en France en 2006 [10]. Celui-ci a vu le jour à la suite de la publication de l'arrêté du 2 août 2005 autorisant à nouveau le prélèvement d'organes et de tissus « sur une personne présentant un arrêt cardiaque et respiratoire persistant " [11]. Conformément au décret, les établissements volontaires pour réaliser ce type de prélèvement doivent signer une convention les engageant à suivre les protocoles validés par l'Agence de la biomédecine, notamment en termes de moyens matériels et humains, de respect du protocole médical et de transmission des données de suivi à des fins d'évaluation. Le premier prélèvement rénal de ce type, basé sur le protocole élaboré par l'Agence de la biomédecine, a eu lieu en octobre 2010. L'objectif de l'Agence est d'augmenter le recours à cette source de greffons afin de permettre un plus grand nombre de malades d'être transplantés [9]. TEG1 Vivre normalement avec un seul rein est tout à fait possible. Une personne majeure vivante, volontaire et en bonne santé peut ainsi donner un rein dans les conditions définies par la loi. Pour répondre aux attentes des patients et de leurs familles et favoriser ce type de greffe, la loi de bioéthique du 7 juillet 2011 a élargi le cercle des donneurs vivants d'organes qui peuvent être le père ou la mère et, par dérogation, un fils ou une fille, un frère ou une sœur du receveur, son conjoint, ses grands-parents, oncles ou tantes, cousins germains et cousines germaines, ainsi que le conjoint du père et de la mère [12]. Peut également être donneur une personne apportant la preuve d'une vie commune d'au moins deux ans avec le receveur ou la preuve d'un lien affectif étroit et stable depuis au moins deux ans avec lui [9]. Le don d'organes de son vivant n'est possible que pour la greffe rénale, hépatique ou du lobe pulmonaire [12].

TEG1 Le nombre de greffes rénales réalisées en France à partir de donneurs vivants augmentait progressivement depuis le début des années 2000 mais reste encore trop modeste et a chuté en 2018 passant à 537 patients greffés par rapport aux 611 l'année précédente. Certaines études ont pourtant montré que les risques encourus pour le donneur demeuraient très faibles, à la fois à court, moyen et long terme. Les résultats pour le receveur se révèlent quant à eux très sensiblement supérieurs à ceux des greffes réalisées à partir de donneurs décédés [13].

Encadré

\section{Consentement présumé du donneur}

En France, la loi dispose que toute personne est considérée comme consentante au don d'éléments de son corps en vue de greffe si elle n'a pas manifesté d'opposition de son vivant [14]. La loi prévoit donc la possibilité de s'opposer par deux moyens : l'inscription au registre national des refus [15] et/ou la communication de sa position à ses proches afin qu'ils en témoignent en cas de décès brutal. En effet, au moment où un prélèvement est envisagé, la loi exige que les équipes médicales s'adressent aux proches pour recueillir l'opposition éventuellement exprimée par le défunt de son vivant.

Sur 1 colonne

TEG1 La liste nationale d'attente pour une greffe rénale comprend l'information du patient et l'étude attentive de son dossier médical qui oriente le choix des examens complémentaires nécessaires. L'information du patient doit s'efforcer d'être la plus objective possible quant aux différentes possibilités de la transplantation. Il est nécessaire de tenir compte des avantages (qualité de vie, liberté de se déplacer, de planifier une grossesse, prolongation de la survie), mais aussi des 
risques généraux (échecs possibles, complications des traitements immunosuppresseurs à court et long terme) et plus spécifiques (risque de récidive de la néphropathie initiale, mortalité et morbidité cardiovasculaire chez les patients les plus âgés). Le dossier médical du patient est étudié soigneusement par l'équipe médico-chirurgicale de transplantation pour préciser la stratégie chirurgicale et définir le traitement immunosuppresseur. À la suite de cette évaluation très complète, le patient est inscrit sur la liste nationale d'attente gérée par l'Agence de la biomédecine ; cette inscription est obligatoire pour pouvoir entrer dans le programme de transplantation [5]. En 2016, 5181 nouveaux malades ont été inscrits sur la liste nationale d'attente pour une greffe rénale, soit une progression des inscriptions qui se poursuit $++7 \%$ en un an, soit 13431 personnes inscrites au total) [8]. La pénurie en greffe rénale s'est discrètement aggravée ces dix dernières années. La durée d'attente varie significativement selon le groupe sanguin ; elle est plus importante pour les patients du groupe B.

TEG1 Les greffons provenant de donneurs décédés sont attribués aux patients inscrits sur la liste d'attente en fonction d'un score composé de différents facteurs : le temps d'attente sur la liste et le délai depuis la mise en dialyse, la compatibilité entre le donneur et le receveur ainsi qu'un indice de facilité à la greffe. Ce score est utilisé par toutes les régions françaises mais le poids accordé à chaque facteur peut varier d'un territoire à l'autre. II existe, en plus, des priorités nationales (pédiatrie, greffe combinée foie-rein ou cœur-rein, parfaite compatibilité entre donneur et receveur) et régionales. Ce système d'attribution complexe, géré par l'Agence de la biomédecine par le biais d'un programme informatique, évolue régulièrement dans un souci d'équité et d'efficacité [5].

Fau_illus1

\section{T1 La procédure de transplantation}

TEG1 Une fois le receveur attribué et le donneur prélevé, la greffe rénale peut commencer. Avant l'intervention, le receveur reçoit un traitement immunosuppresseur par voie intraveineuse (IV). Une antibiothérapie prophylactique est également instaurée avant l'incision de la peau de ce dernier. TEG1 La transplantation rénale est effectuée en limitant le temps d'ischémie froide (délai entre le clampage artériel chez le donneur et le déclampage artériel chez le receveur, pendant lequel le rein n'a plus de circulation sanguine). En attendant, le greffon est conservé dans la majorité des cas au froid après avoir été perfusé par un liquide de conservation ou, technique plus récente, grâce à des machines à perfusion pulsatile [5]. Les données sur l'utilisation de la perfusion hypothermique pulsatile pour les reins au cours de la préservation des organes sont limitées. Une étude réalisée aux États-Unis en 2008 a montré qu'elle était sûre et conduisait à une amélioration de la fonction du greffon, en particulier pour les reins marginaux à partir de critères étendus ou des donneurs décédés. Les effets à long terme sur la survie du greffon restent à évaluer [16].

TEG1 Si le donneur est vivant, le rein est prélevé immédiatement avant la transplantation, par voie classique ou cœliochirurgie. II ne subit qu'une ischémie tiède.

TEG1 Le rein est implanté dans la fosse iliaque droite dans la plupart des cas. Les anastomoses vasculaires (connexion entre deux vaisseaux) sont effectuées sur les vaisseaux iliaques, tandis que I'uretère est réimplanté sur la vessie ou sur l'uretère du receveur. Dès que les anastomoses vasculaires sont réalisées, le patient reçoit un remplissage vasculaire abondant couplé à une injection de furosémide (diurétique hypokaliémant de l'anse de Henlé) au moment du déclampage, de manière à favoriser une reprise rapide de la diurèse. La durée d'intervention est de deux à trois heures.

TEG1 Le lever du patient greffé est possible le deuxième jour, ainsi que la reprise de l'alimentation. Ce dernier peut quitter l'hôpital après environ deux à trois semaines. Toutefois, il doit rester sous 
surveillance médicale très régulière et réaliser des examens de laboratoire très rapprochés, une ou deux fois par semaine pendant les trois premiers mois, puis à intervalles de plus en plus espacés. Cette surveillance rigoureuse est nécessaire pour l'établissement d'un diagnostic précoce et précis des diverses complications, ces dernières pouvant contrarier l'évolution de la transplantation à court, moyen et long terme, ainsi que le traitement instauré. Elle est donc indispensable et peut être effectuée soit par le centre de transplantation, soit en collaboration avec le néphrologue et le médecin traitant [5].

\section{Points à retenir}

- En 2016, 46872 patients en insuffisance rénale chronique dialysés et 37800 greffés rénaux étaient dénombrés en France.

- En 2018, 3546 patients ont reçu une greffe rénale, dont 537 à partir de donneurs vivants, en légère baisse par rapport aux deux années précédentes.

- Le rein est l'organe le plus souvent greffé en France.

- Les greffons provenant de donneurs décédés ou vivants sont attribués aux patients inscrits sur une liste d'attente gérée par l'Agence de la biomédecine.

- La seule contre-indication absolue à la greffe de rein est l'existence d'un cancer métastasé.

Sur 1 colonne après la puce de fin

\section{Références}

[1] Caille Y, Derayb G, Isnard Bagnisb C. Les Français connaissent mal leurs reins... Résultats d'une enquête sur les maladies rénales réalisée auprès du grand public. Néphrologie et Thérapeutique. 2006;3:55-9.

[2] Noël LH. Atlas de pathologie rénale. Paris: Médecine-Sciences Flammarion; 2008.

[3] Suc JM, Durand D. Manuel de néphrologie clinique. Paris: Ellipses; 2011.

[4] Institut national de la santé et de la recherche médicale (Inserm). Insuffisance rénale. www.inserm.fr/information-en-sante/dossiers-information/insuffisance-renale

[5] Legendre C. Maladie rénale chronique. Rev Prat. 2012;62(1):27-75.

[6] Bongard V, Dallongeville J, Arveiler D et al. Estimation et caractérisation de l'insuffisance rénale chronique en France. Annales de cardiologie et d'angéiologie. 2012;61(4):239-44.

[7] Grunfeld JP. Les essais thérapeutiques en néphrologie, du concept à la pratique. Boulogne-

Billancourt: Vivactis Medical Éducation; 2007.

[9] Agence de la biomédecine. www.dondorganes.fr/

[8] Agence de la biomédecine. Le rapport médical et scientifique du prélèvement et de la greffe en France. www.agence-biomedecine.fr/annexes/bilan 2016/donnees/organes/06-rein/pdf/rein.pdf

[10] Cabrol C. Prélèvements d'organes sur donneur à cœur arrêté. Paris: Académie nationale de médecine; 2007. www.academie-medecine.fr/Upload/anciens/rapports_317_fichier_lie.rtf 
[11] Arrêté du 2 août 2005 fixant la liste des tissus et des cellules pour lesquels le prélèvement sur une personne décédée présentant un arrêt cardiaque et respiratoire persistant est autorisé. Journal officiel de la République française du 6 août 2005.

www.legifrance.gouv.fr/affichTexte.do?cidTexte=JORFTEXT000000262916

[12] Loi n 2011-814 du 7 juillet 2011 relative à la bioéthique.

www.legifrance.gouv.fr/affichTexte.do?cidTexte=JORFTEXT000024323102

[13] Agence de la biomédecine, Conseil d'orientation. Délibération 2010-CO-45. La greffe rénale à partir de donneurs vivants. www.agence-biomedecine.fr/IMG/pdf/greffe_renale_08102010.pdf

[14] Code la santé publique. Article L1232-1.

www.legifrance.gouv.fr/affichCodeArticle.do?cidTexte=LEGITEXT000006072665\&idArticle=LEGIARTIO 00031931933\&dateTexte=\&categorieLien=id

[15] www.registrenationaldesrefus.fr

[16] Matsuoka L, Almeda J, Mateo R. Pulsatile perfusion of kidney allografts. Current opinion in Organ Transplantation. 2009;14(4):365-9.

Déclaration de liens d'intérêts

Les auteurs déclarent ne pas avoir de liens d'intérêts.

\section{Illustration}

\section{Fau_illus1}

Lorsque le donneur est vivant, le rein ne subit qu'une ischémie tiède, car il est prélevé immédiatement avant la transplantation, par voie classique ou cœliochirurgie.

(C) A. Noor/BSIP

Sur 1 colonne près de son appel 


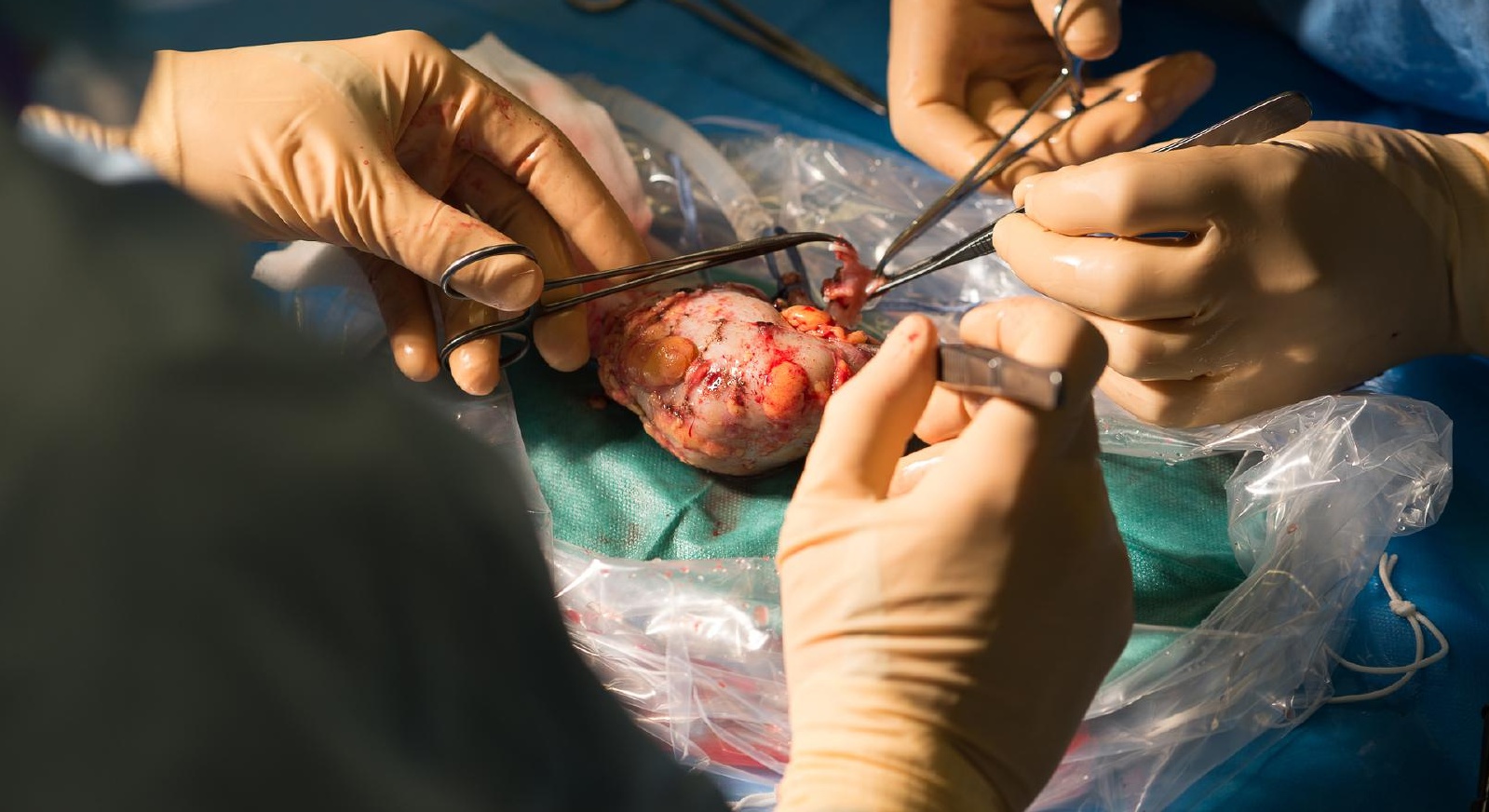

